\title{
Optimal Spectrum Sensing Time and Transmit Power Based on Energy Efficiency in Cognitive Radio Networks
}

\author{
J.X. Dai \\ School of Science \\ Nanjing University of Posts and Telecommunications \\ Nanjing, China \\ J.W. Liang \\ College of Telecommunications \&Information Engineering \\ Nanjing University of Posts and Telecommunications \\ Nanjing, China
}

\author{
Z.J. Ni \\ College of Telecommunications \&Information Engineering \\ Nanjing University of Posts and Telecommunications \\ Nanjing, China \\ X. Wang \\ School of Science \\ Nanjing University of Posts and Telecommunications \\ Nanjing, China
}

\author{
T. Yuan \\ College of Telecommunications \&Information Engineering \\ Nanjing University of Posts and Telecommunications \\ Nanjing, China
}

\begin{abstract}
The optimization problems of spectrum sensing time and power in CR network are investigated in this paper. Firstly, the energy efficiency optimization problem, whose variables that should be jointly optimized are transmit power and sensing time of cognitive users, is derived. Then, the relations between the energy efficiency and transmit power, the energy efficiency and spectrum sensing time are analyzed, respectively. The numerical results show that $C R$ network can achieve almost the maximum achievable data rate with significant energy saving through the joint optimization.
\end{abstract}

Keywords- cognitive radio; spectrum sensing; energy efficiency; sensing time; transmit power

\section{INTRODUCTION}

Recently, Cognitive radio (CR) is an efficient way to improve spectrum utilization [1-3]. The basic idea of CR is to allow unlicensed or secondary users (SU) to access the licensed spectrum originally allocated to primary users (PU) without reducing the quality-of-service (QoS) of the PU. On the other hand, rapidly rising energy costs and increasingly rigid environmental standards have led to an emerging trend of addressing "energy efficiency" aspect of wireless communication technologies [4].Improving energy efficiency is the ability to reduce environmental pollution and the waste of resources. It is a new communication concept which will achieve harmony between man and nature and the sustainable development.

- User --- Extend the usage time of mobile phones and other mobile devices

- Operators --- Reduce operators' operational overhead and ease the crisis of the environment end energy

- Environment --- Reduce the impact on the environment, carbon dioxide emissions and electromagnetic radiation

The energy-efficient transmission that takes into account the energy consumption in CR nets is very important to batterypowered devices since they are energy limited. The power consumption of the circuit contains the power consumption of the circuit at the transmitting end and the receiving end [5-6]. Although we can improve power efficiency, the spectrum efficiency fells. However we improve the spectrum efficiency, but power efficiency decreases. In the previous researches, the tradeoff between throughput and sensing time was addressed in [7] for a single frequency band, where the authors studied the problem of optimal sensing time that maximizes the throughput under a constraint on the probability of detection. However, the power allocation was not considered. This work was extended in [8] [9] for a wideband spectrum, where the problem of finding the optimal sensing time and power allocation scheme that maximizes the average achievable throughput was studied under two different power constraints. In this paper, we will find a balance between them to solve the problem based on energy efficiency, which is quantified by the persistence function, throughput $/ P_{\text {total }}$. The objective of this paper is to design the spectrum sensing time together with transmit power of SU to maximize the energy efficiency. 


\section{OPTIMIZATION OF SPECTRUM SENSING PARAMETER BASED ON ENERGY EFFICIENCY}

\section{A. Secondary User's Operating Mode}

Figure I [10] shows secondary user's operating mode. Before users transmit the data, secondary user should detect the channel of primary user in simple energy detection method [11]. If the channel is idle, secondary user can conduct data transmission. During the process of data transmission, secondary user will detect the channel periodically for avoiding interference to the primary user. $T_{c}$ is the interval between two consecutive channel detection. After the circulation of sensing, if the primary user's channel is found busy, secondary user must stop data transmission to keep waiting for the next result of next detection.

In this paper, we consider that the single secondary user sense spectrum according to the frame structure. Secondary user access based on overlay mode. We assume that secondary user might just sense one channel every frame with the white Gaussian noise. We also assume that the probability of busy channel obeys the negative exponential distribution of parameters $\alpha$, probability of busy channel obeys the negative exponential distribution of parameters $\beta$.Then we can come to conclusion based on queuing theory.

$$
\begin{gathered}
P_{\text {on }}=\frac{\beta}{\alpha+\beta} \\
P_{\text {off }}=\frac{\alpha}{\alpha+\beta}
\end{gathered}
$$

where $P_{\text {on }}$ represents the probability of busy channel, $P_{\text {off }}$ represents the probability of idle channel.

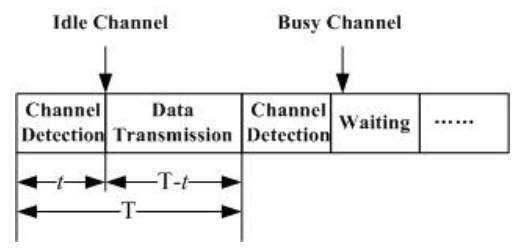

FIGURE I. SINGLE PU CHANNEL.

\section{B. The Probability of Detection And False-Alarm}

The main indicators to measure the performance of spectrum sensing are detecting probability $P_{d}$ [12], false alarm probability $P_{f}$. The probability of false-alarm determines the percentage of the white spaces that are misclassified as occupied. When $P_{f}$ increases, it means that there are less opportunistic spectrum access.

Cognitive users detect periodically whether the primary user signal is present every frame. Spectrum sensing for opportunistic access may be formulated as a binary hypothesis test.

$$
r(t)=h x(t)+n(t)
$$

where ${ }^{x(t)}$ is the primary users' transmitted signal, $n(t)$ is the additive white Gaussian noise (AWGN) and $h$ is the amplitude gain of the channel. In this paper, simple energy detection is chosen as the underlying detection scheme [14]. $P_{d}(\lambda, t)$ and $P_{f}(\lambda, t)$ may be expressed as:

$$
\begin{aligned}
& P_{d}(\lambda, t)=\bar{P}_{d}(\lambda, t) P_{\text {on }} \\
& P_{f}(\lambda, t)=\bar{P}_{f}(\lambda, t) P_{\text {off }}
\end{aligned}
$$

where $\bar{P}_{d}(\lambda, t)$ represents the detecting probability by using energy detection, $\bar{P}_{f}(\lambda, t)$ represents false alarm probability, $t$ is sensing time[13]

$$
\begin{gathered}
\bar{P}_{d}(\lambda, t)=Q\left[\left(\lambda-t f_{s}-\gamma\right) / \sqrt{2 t f_{s}+4 \gamma}\right] \\
\bar{P}_{f}(\lambda, t)=Q\left[\left(\lambda-t f_{s}\right) / \sqrt{2 t f_{s}}\right]
\end{gathered}
$$

Where $t$ represents the sensing time, $\lambda$ denotes the sensing threshold of energy detector, $\gamma$ is the received signalto-noise ratio (SNR) from the PU measured at the secondary receiver, $f_{s}$ represents the sampling frequency and finally $Q(x)$ is Gaussian function

$$
Q(x)=\frac{1}{\sqrt{2 \pi}} \int_{x}^{\infty} e^{-\frac{u^{2}}{2}} d u
$$

\section{CR User's Throughput and Power}

When Bias risks identified and interference rate controlled below the threshold, the secondary users' average throughput can be expressed as :

$$
R(\lambda, T, t)=\frac{C\left[1-\bar{P}_{f}(\lambda, t)\right] P_{\text {off }}(T-t)}{T}
$$

where $C$ is channel capacity, $T$ is the length of one frame.

The power of transmission, sensing and the circuit consumption need to be considered in a real system. $P_{s}$ is the sensing power. The transmit power during spectrum sensing time units can be expressed as $P_{T}=\leq P_{T, \text { max }}$. Where $P_{T, \text { max }}$ is the maximum transmit power. The power of circuit consumption contains $P_{c, \mathrm{Tx}}$ (at the transmitting end) and $P_{c, \mathrm{Re}}$ (at the receiving end). We set the $P_{c}$ is a fixed value, $P_{c}$ can be expressed as

$$
P_{c}=P_{c, \mathrm{Tx}}+P_{c, \mathrm{Re}}
$$

When the channel is indeed idle (i.e., the estimation is correct), the secondary user is assigned a portion of the channel at a certain transmit power during the transmission time. The average power can be expressed as 


$$
\bar{P}=\frac{t P_{s}+P_{c}+(T-t) P_{t}\left[1-\bar{P}_{f}(\lambda, t)\right] P_{\text {off }}}{T}
$$

D. A System Model Based on the Energy Efficiency Optimization problem can be modelled as

$$
\begin{array}{ll}
\max _{t, P_{t}} & \frac{C\left[1-\bar{P}_{f}(\lambda, t)\right] P_{\text {off }}(T-t)}{t P_{s}+P_{c}+(T-t) P_{t}\left[1-\bar{P}_{f}(\lambda, t)\right] P_{\text {off }}} \\
\text { s.t. } & P_{T, \text { min }} \leq P_{t} \leq P_{T, \max } \\
& 1 \leq t \leq T
\end{array}
$$

where $C=\log _{2}\left(1+g P_{t} / \sigma_{n}^{2}\right)$.Hence (12) can be written as

$$
\begin{array}{ll}
\max _{t, P_{t}} & \log _{2}\left(1+g P_{t} / \sigma_{n}^{2}\right)\left[1-\bar{P}_{f}(\lambda, t)\right] P_{\text {off }}(T-t) \\
\text { s.t. } & P_{s}+P_{c}+(T-t) P_{t}\left[1-\bar{P}_{f}(\lambda, t)\right] P_{\text {off }} \\
& 1 \leq t \leq T
\end{array}
$$

\section{ANALYSIS OF THE OPTIMAL MODEL}

A. Relation between the Energy Efficiency and Transmit Power

Assume the number of sensing time is fixed. The problem (13) can be simplified as

$$
\left\{\begin{array}{l}
\max _{P_{t}} F\left(P_{t}\right)=\max _{P_{t}} \frac{\log _{2}\left(1+b_{1} P_{t}\right)}{P_{t}+b_{2}} \\
\text { s.t. } 0 \leq P_{t} \leq P_{T, \text { max }}
\end{array}\right.
$$

where

$$
b_{1}=\frac{g}{\sigma_{n}^{2}}, \quad b_{2}=\frac{t P_{s}+P_{c}}{(T-t)\left[1-\bar{P}_{f}(\lambda, t)\right] P_{\text {off }}}
$$

Theorem 1.The optimal transmit power $P_{t, \text { opt }}$ exists uniquely and satisfies

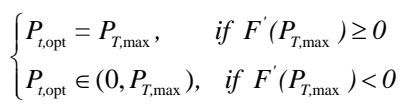

Proof: Because $F\left(P_{t}\right)$ is continuous when $P_{t} \geq 0$, the derivative is

$$
F\left(P_{t}\right)=\frac{\frac{b_{1}}{\ln 2} \frac{P_{t}+b_{2}}{b_{1} P_{t}+1}-\log _{2}\left(b_{1} P_{t}+1\right)}{\left(P_{t}+b_{2}\right)^{2}}
$$

Let

$$
\begin{aligned}
& g\left(P_{t}\right)=\frac{b_{1}}{\ln 2} \frac{P_{t}+b_{2}}{b_{1} P_{t}+1}-\log _{2}\left(b_{1} P_{t}+1\right) \\
& q\left(P_{t}\right)=\left(P_{t}+b_{2}\right)^{2}
\end{aligned}
$$

Then

$$
g^{\prime}\left(P_{t}\right)=\frac{-b_{1}^{2}\left(P_{t}+b_{2}\right)}{\ln 2\left(b_{1} P_{t}+1\right)^{2}}<0
$$

and

$$
\begin{gathered}
g(0)=\frac{b_{1} b_{2}}{\ln 2}>0 \\
\lim _{P_{t} \rightarrow \infty} g\left(P_{t}\right)<0
\end{gathered}
$$

Thus $g\left(P_{t}\right)$ is monotone decreasing. In addition, $q\left(P_{t}\right)$ is always positive and monotone increasing.

We have

$$
F^{\prime}(0)=\frac{b_{1}}{b_{2} \ln 2}>0 \quad \lim _{P_{t} \rightarrow \infty} F^{\prime}\left(P_{t}\right)<0
$$

If $g\left(P_{T, \max }\right) \geq 0$, then $g\left(P_{t}\right)$ is always positive in[0, $P_{T, \text { max }}$ ].So $F^{\prime}\left(P_{t}\right)$ is always positive $\operatorname{in}\left[0, P_{T, \max }\right]$, which means $F\left(P_{t}\right)$ is monotone increasing in $\left[0, P_{T, \max }\right]$. Thus the optimal $P_{t}$ is $P_{T, \max }$.

If $g\left(P_{T, \max }\right)<0$, then $g\left(P_{t}\right)$ is positive first then negative as $P_{T}$ increases in $\left[0, P_{T, \max }\right]$,so is $F\left(P_{t}\right)$, which means $F\left(P_{t}\right)$ is monotone increasing first then monotone decreasing in $[0$, $\left.P_{T, \text { max }}\right]$. Thus $P_{t, \text { opt }}$ should be in( $\left.0, P_{T, \max }\right)$.

Evidently, $P_{t, \text { opt }}$ is unique for both cases.

B. Relation between the Energy Efficiency and Sensing Time

Assume the transmit power is fixed. To simplify analysis, we denote

$$
\begin{gathered}
W(t)=\log _{2}\left(1+g P_{t} / \sigma_{n}^{2}\right)\left[1-\bar{P}_{f}(\lambda, t)\right] P_{\text {off }}(T-t) \\
\psi(t)=t P_{s}+P_{c}+(T-t) P_{t}\left[1-\bar{P}_{f}(\lambda, t)\right] P_{\text {off }}
\end{gathered}
$$

The problem (13) can be expressed as

$$
\begin{aligned}
& \max _{t} \frac{W(t)}{\psi(t)} \\
& \text { s.t. } 1 \leq t \leq T
\end{aligned}
$$

To solve the above question, we can use the fractional optimization in [15] for reference, define a new function

$$
f(t, \tau)=W(t)-\tau \psi(t)
$$

where $\tau$ is an arbitrary positive number. Define another function

$$
s(\tau)=\max _{t} f(t, \tau)
$$

We can draw conclusions as follows:

Theorem 2. If it is a decreasing function of $s(\tau)$, (23) will get its optimal solution at the point $s(\tau)=0$.

Proof: First, prove that $s(\tau)$ is a decreasing function of $\tau$. 
$\psi(t)>0$ for any given $t$. Hence, for a given $t, f(t, \tau)$ decreases with increase of $\tau$.

$s(\tau)=\max _{t} f(t, \tau) \geq f(t, \tau)>f(t, \tau+\varepsilon), \forall t$

where $\varepsilon$ is an arbitrary number larger than 0 . We have

$$
s(\tau)>\max _{t} f(t, \tau+\varepsilon)=s(\tau+\varepsilon)
$$

$s(\tau)$ is a decreasing function of $\tau$.

Second, prove that question (23) gets its optimal solution at the point $s(\tau)=0$.

For a given ${ }^{t}$, we have $f(t, \eta(t))=0$. Suppose that $t_{\text {opt }}$ is the optimal solution of (23). Since $\eta\left(t_{\mathrm{opt}}\right)$ is the maximum value of energy efficiency, $\tau=\eta\left(t_{\mathrm{opt}}\right)$ is the maximum value of $\tau$ such that $f(t, \tau)=0$.

Then, for a given $\bar{\tau}$ such that $s(\bar{\tau})=0$ and $\bar{t}=\arg \left\{\max _{t} f(t, \bar{\tau})\right\}$. Suppose $\tilde{\tau}>\bar{\tau}$, since $s(\tau)$ is a monotone decreasing function, for an arbitrary number $t$, we have $s(\tilde{\tau})<0$. Hence $\bar{\tau}$ is the maximum value such that $f(t, \tau)=0$. So that $\bar{\tau}=s\left(t_{\mathrm{opt}}\right)$ and $\bar{t}=t_{\mathrm{opt}}$.

As we mentioned before, $s(\tau)$ is a monotone decreasing function, we can find the $\bar{\tau}$ such that $s(\bar{\tau})=0$ by dichotomy method.

Theorem 3. If $\tau$ has been given and $s(\tau) \geq 0$, in the given $t$ interval $[1, T), f(t, \tau)$ is a convex function. $t$

Proof: First, we find the second derivative of $f(t, \tau)$ over

$$
\frac{\partial^{2} f(t, \tau)}{\partial t^{2}}=\left(C P_{\text {off }}-\tau P_{t}\right) \bar{P}_{f}^{\prime}(\lambda, t)-\left(C P_{\text {off }}-\tau P_{t}\right)(T-t-1) \bar{P}_{f}^{\prime \prime}(\lambda, t)
$$

If $P_{f}(t)<0.5, P_{f}(t)$ is a decreasing function[7]. It is a concave function, it means

$$
P_{f}^{\prime}(t)<0 \text { and } P_{f}^{\prime \prime}(t)>0 \text {. }
$$

Since $s(\tau) \geq 0$, then $C P_{\text {off }}-\tau P_{t}>0$, and we get $f^{\prime \prime}(t, \tau)<0$.

\section{Simulation Results AND Discussion}

In this section, we present the numerical results of the energy efficiency of the CRN. of CR

The dependence of energy efficiency on the transmit power

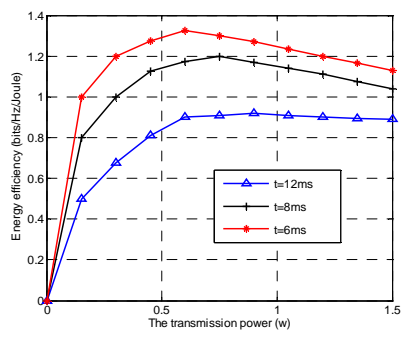

FIGURE II . THE DEPENDENCE OF ENERGY EFFICIENCY ON TRANSMIT POWER OF CR $\left({ }^{{ }_{t}}\right)$ GIVEN SENSING TIME T=6MS (RED ASTERISKS), $\mathrm{T}=8 \mathrm{MS}$ (BLACK PLUS) AND $\mathrm{T}=12 \mathrm{MS}$ (BLUE TRIANGLES). THE PARAMETERS ARE B=3MHZ, T=20ms, $P_{\text {off }}=0.7$, $P_{c=0.01 \mathrm{w},} P_{s=0.05 \mathrm{w}, \mathrm{g}=0.1,} P_{d=0.9,} P_{f=1-} P_{d=0.1}$.

Figure II displays energy efficiency as a function of transmit power of CR $\left({ }^{P_{t}}\right)$ given sensing time ${ }^{t}=6 \mathrm{~ms}$ (red asterisks), ${ }^{t}=8 \mathrm{~ms}$ (black plus) and ${ }^{t}=12 \mathrm{~ms}$ (blue triangles). When $P_{t}$ is small, energy efficiency increases sharply with increasing $P_{t}$, whereas as $P_{t}$ gets larger, energy efficiency decreases slowly. As a whole, an optimal transmit power of CR exists subject to the highest energy efficiency. Moreover, comparing different sensing time, our results about energy efficiency indicates that with smaller sensing time at constant transmit power, energy efficiency gets larger.

The dependence of energy efficiency on sensing time

FigureIIIdisplays energy efficiency as a function of sensing time t given transmit power of CR: ${ }^{P_{t}}=0.2 \mathrm{w}$ (red asterisks), $P_{t}=0.5 \mathrm{w}$ (black plus) and $P_{t}=0.8 \mathrm{w}$ (blue triangles). When sensing time ${ }^{t}$ is small, we will suffer from high false alarm and miss the transmission opportunities. Therefore, throughput is reduced and energy efficiency is also reduced. When sensing time ${ }^{t}$ is increased to certain value, the reduced false alarm and therefore higher throughput may not offset the increasing energy consumption for spectrum sensing. Hence, energy efficiency will drop. As a whole, an optimal sensing time t exists subject to the highest energy efficiency. Secondly, it is observed that optimal sensing time is increased with the increasing transmit power.

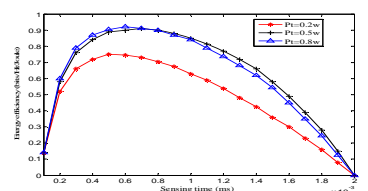

FIGURE III. THE DEPENDENCE OF ENERGY EFFICIENCY ON SENSING TIME T GIVEN TRANSMIT POWER OF CR $P_{t}=0.2 \mathrm{w}$ (RED ASTERISKS), $P_{t}=0.5 \mathrm{w}$ (BLACK PLUS) AND $P_{t}=0.8 \mathrm{w}$ (BLUE TRIANGLES). THE PARAMETERS ARE B=3MHZ, T=20ms, $P_{\text {off }}=0.7$, $P_{c}=0.01 \mathrm{w}, \quad P_{s}=0.05 \mathrm{w}, \mathrm{g}=0.1$. 


\section{CONCLUSION}

In this paper, the optimization problems of spectrum sensing and power allocation based on energy efficiency in CR network were investigated. There was a trade-off between throughput and power, throughput and sensing time when energy efficiency was chosen as the measurement. The simulation results show the effectiveness of the proposed algorithm and the necessity of the energy efficiency function chosen as the measurement.

\section{ACKNOWLEDGMENT}

This work is supported by STITP of Nanjing University of Posts and Telecommunications (No. SZDG 2014022).

\section{REFERENCES}

[1] FCC, ET Docket No 03-237 Notice of inquery and notice of proposed Rulemarking, November 2003..

[2] Ghasemi, Amir, and Elvino S. Sousa, "Spectrum sensing in cognitive radio networks: requirements, challenges and design trade-offs,” IEEE Communications Magazine, 46(4) , pp. 32-39. 2008

[3] Neel J O D. "Analysis and design of cognitive radio networks and distributed radio resource management algorithms” [D]. Virginia Polytechnic Institute and State University, 2006.

[4] F. R. Yu, X. Zhang, and V. C. M. Leung, "Green Communications and Networking”. New York: CRC Press, 2012

[5] Haykin S. "Cognitive radio: brain-empowered wireless communications.” Selected Areas in Communications, IEEE Journal on, 23(2), pp. 201-220, 2005.

[6] Akyildiz I F, Lee W Y, Vuran M C, et al. "Next generation/dynamic spectrum access/cognitive radio wireless networks: a survey,” Computer Networks, 50(13), pp. 2127-2159, 2006

[7] Liang Y C, Zeng Y, Peh E C Y, "Sensing-throughput tradeoff for cognitive radio networks," IEEE Transactions on Wireless Communications,vol. 7, no. 4, pp. 1326-1337, 2008

[8] Pei Y, Liang Y C, Teh K, "How much time is needed for wideband spectrum sensing?,” IEEE Transactions on Wireless Communications, vol. 8, no. 11, pp. 5466-5471, 2009

[9] Stotas S, Nallanathan A, "Optimal sensing time and power allocation in multiband cognitive radio networks ," IEEE Transactions on Communications, vol. 59, no. 1, pp. 226-235, 2011.

[10] Ghasemi A, Sousa E S. "Collaborative spectrum sensing for opportunistic access in fading environments"[C]//New Frontiers in Dynamic Spectrum Access Networks, 2005. DySPAN 2005. 2005 First IEEE International Symposium on. IEEE, 2005: 131-136.

[11] H. Urkowitz, "Energy detection of unknown deterministic signals, "Proceedings of IEEE, vol. 55, pp. 523-231, April 1967

[12] Hurt G, Sullivan T, Patrick G, et al. Interference Protection Criteria: Phase 1, Compilation from Existing Sources. US Department of Commerce, National Telecommunications and Information Administration, 2005..

[13] Pei Y, Liang Y C, Tech, KC, "Energy-efficient design of sequential channel sensing in cognitive radio networks: optimal sensing strategy, power allocation, and sensing order," IEEE Journal on Selected Areas in Communications, 2011, 29(8): 1648-1659.

[14] Shi, Z., K. Tech, "Energy-efficient joint design of sensing and transmission durations for protection of primary user in cognitive radio systems”, IEEE Global Telecommunications Conference, pp. 1-4, 2011.

[15] Peh, Liang Y C, "Energy-Efficient Cooperative Spectrum Sensing in Cognitive Radio Networks”, IEEE Global Telecommunications Conference, pp. 1-5, 2011 